\title{
WRS/ WARISATA
}

www. revistawarisata.org

Volumen 3 / Nro. 7 / Enero - abril 2021 / ISSN: 2708 - 6305 / ISSN-L: 2708 - 6305 / Pág. 40 - 52

\section{Aprendizaje por descubrimiento aplicado a la multiplicación de números naturales}

\author{
Applied discovery learning to the multiplication of natural numbers
}

\author{
- Brenda Maritza Barrios Villarreal \\ brendabarrios0411@gmail.com \\ Código ORCID: 0000-0002-2728-1952
}

Ministerio del Poder Popular para la Educación, Caracas, Venezuela

\author{
Efraín Eduardo Camacho Hernández \\ efedcahe@hotmail.com \\ Código ORCID: 0000-0002-6859-2431
}

Universidad de Carabobo, Valencia, Venezuela

I Artículo recibido en octubre 2020 | Arbitrado en noviembre 2020 | Aceptado en diciembre 2020 I Publicado en enero 2021

\section{RESUMEN}

Palabras clave:

Evaluación;

Aprendizaje;

Descubrimiento.
La investigación tuvo por objetivo general analizar el aprendizaje por descubrimiento aplicado a la multiplicación de números naturales en los estudiantes del quinto grado de la Unidad Educativa Estadal Aragüita. Como fundamentos se consideraron las teorías del aprendizaje significativo de Ausubel, motivación de Maslow y aprendizaje cooperativo de Vygotsky. Se adscribe a una investigación de campo no experimental cuya muestra fue de carácter no probabilística constituida por 26 estudiantes del quinto grado a quienes se les aplicó una Lista de Cotejo conformada por 17 ítems, para determinar el grado o nivel de aprendizaje en la multiplicación de números naturales. La validez del instrumento se realizó a través del juicio de expertos y la confiabilidad se realizó mediante el coeficiente Kuder-Richardson, la cual fue de 0,85 resultando altamente confiable. Para el cierre de esta investigación, se dilucidó la ausencia de aplicación de teorías que sustentaran el aprendizaje significativo de los estudiantes.
Keywords:

Assessment; Learning; Discovery

\section{ABSTRACT}

The research had the general objective of analyzing the discovery learning applied to the multiplication of natural numbers in the fifth grade students of the Aragüita State Educational Unit; Ausubel's significant learning, Maslow's motivation, and Vygotsky's cooperative learning were considered among the theories. It is part of a non-experimental field investigation whose sample was of a non-probabilistic nature made up of 26 fifth grade students; to whom a checklist made up of 17 items was applied to determine the degree or level of learning in the multiplication of natural numbers. The validity of the instrument was carried out through expert judgment and reliability was performed using the Kuder-Richardson coefficient, which was 0.85 , resulting highly reliable. For the closing of this investigation, the absence of application of theories that support the significant learning of the students was elucidated. 


\section{INTRODUCCIÓN}

A lo largo de la historia la educación ha sido un factor esencial para la adquisición y el manejo de conocimientos e información entre diferentes grupos sociales. En todo momento se aprende y se generan conocimientos nuevos con lo que cada individuo propicia su desarrollo personal, social y profesional, generando así un aprendizaje por descubrimiento donde la creatividad, la destreza y la solución de problemas, son elementos primordiales.

Por esta razón, la presente investigación sostiene que el docente debe orientar el aprendizaje de sus estudiantes a partir de esos conocimientos previos en determinadas áreas del conocimiento, desde una perspectiva de la cotidianidad, es decir, desde su propio contexto, y que esto les ayude a su vez a comprender otros contextos. Al respecto, Delors (1996) señala que los pilares fundamentales de la educación son: aprender a ser, aprender a conocer, aprender a vivir juntos y aprender a hacer. También habla sobre las teorías que fundamentan el modelo curricular y la capacitación docente, tomando en cuenta tópicos como el aprendizaje constructivista de Piaget, el aprendizaje mediano de Feurestein, el aprendizaje por descubrimiento de Bruner, la teoría potencial del aprendizaje de Vygotsky y el aprendizaje significativo de Ausubel.

Por lo antes expuesto, se puede decir que la educación es un factor impulsor y transformador para el crecimiento y el desarrollo del educando y por ende, de un país. El sistema educativo venezolano es el ente para ejecutar la planeación requerida en los cambios de la dinámica social actual. Los docentes, en comunión con los estudiantes durante el proceso de enseñanza aprendizaje, orientan las actividades del currículo a través de la evaluación, la coevaluación y la autoevaluación, para detectar y corregir debilidades, enfocando el aprendizaje hacia las teorías y estrategias más adecuadas para el logro de los objetivos y competencias.

En este sentido, el pensamiento pedagógico no debe limitarse a la ideología, teoría o contenidos que fundamentan los diversos elementos del diseño curricular (políticas educativas, programas, planes y libros de texto), como ha sucedido en algunos de los momentos de la educación venezolana, sino que debe materializarse en la práctica. En concordancia con la Ley Orgánica de Educación (2009) en su artículo 14, se señala que: "La educación es un derecho humano y un deber social fundamental concebida como un proceso de formación integral, gratuita, laica, inclusiva y de calidad, permanente, continua e interactiva, promueve la construcción social del conocimiento..." (p.17)

Por lo tanto, la presente investigación pretende mejorar la metodología de enseñanza tradicional, específicamente en el área de matemáticas relacionada con la multiplicación de números naturales, con la finalidad de propiciar un aprendizaje por descubrimiento que permita la obtención de herramientas, habilidades y destrezas significativas, y que a este proceso se le pueda dar continuidad en sucesivas etapas de formación o capacitación.

De este modo, se considera la educación matemática desde el enfoque del Subsistema Educativo Bolivariano (SEB), lo que contribuye significativamente a desarrollar lo metódico, el pensamiento ordenado y el razonamiento lógico. Actualmente se imparte en las aulas una metodología para la enseñanza de la matemática con objetivos aislados de la realidad del estudiante, donde reciben el contenido de forma poco significativa, con resolución de ejercicios rutinarios y conceptos mecanizados. Por esta razón, lo que se buscó con esta investigación fue reformular los contenidos, estrategias, propósitos, recursos y todo aquello relacionado con el proceso de enseñanza y aprendizaje de los estudiantes del quinto grado de la Unidad Educativa 
Estadal "Aragüita". En concordancia con Gascón, Muñoz, Fonseca y Artigue citados en Álvarez (2006):

Las causas de las dificultades presentes en la enseñanza de la matemática se focalizan sobre el estudiante por falta de motivación, insuficiente desarrollo cognitivo, falta de conocimientos previos, lo que genera un alto porcentaje de estudiantes aplazados, deserción escolar, temor e inclusive odio hacia esta ciencia. (p.32)

Como respuesta a esta problemática, muchas instituciones educativas del país y organismos gubernamentales, se han centrado en buscar soluciones efectivas en la enseñanza de la matemática con relación a políticas pedagógicas expuestas en diferentes planes de la nación, como por ejemplo, el Currículo Básico Nacional, donde se señala la importancia de las ideas previas de los estudiantes, las diferencias individuales, las condiciones del contexto, la aplicabilidad de los conocimientos, la consideración del alumno como elemento central del proceso y la adecuación de actividades, su caracterización y finalmente la estimación del importante papel del docente, como parte del proceso de construcción vital del alumno.

Se considera que todos estos aspectos mencionados, son relevantes para el desarrollo e implementación de los objetivos, mediante una estructuración didáctica de los contenidos, tomando en cuenta las necesidades $y$ problemas que afecten al alumno, al maestro, a la institución y a la comunidad. El rol del docente en esta metodología consiste en guiar el aprendizaje de los estudiantes, siempre considerando el contexto en el que se encuentran.

Por lo tanto, la investigación induce a revisar la metodología de cómo se le está enseñando al estudiante los contenidos para propiciar un aprendizaje por descubrimiento, puesto que es un tema de gran importancia y con numerosos antecedentes. Todo esto en concordancia con el Currículo Básico Nacional (1997) donde se indica que:

El Diseño Curricular de este nivel se sustenta en una concepción de la enseñanza y del aprendizaje que se alimenta de los aportes de diversas corrientes psicológicas, asociadas genéricamente a la psicología cognitiva, entre los cuales cabe destacar: la Teoría Genética de Piaget, la Teoría del Aprendizaje Significativo de Ausubel, la Teoría Sociocultural de los Procesos Superiores de Vygotsky. (p. 12)

En este sentido, el aprendizaje por descubrimiento aplicado a la multiplicación de números naturales, en donde el estudiante, a través de la información que recibe en un contexto vinculado con la interacción social y cultural, sea capaz de relacionar, analizar y llegar a un juicio crítico, por medio del cual, encuentre el sentido lógico, práctico y utilitario de lo que está aprendiendo en la escuela.

Para el abordaje de esta problemática educativa, el diseño curricular, tal como lo plantea el MPPE en el Currículo Nacional Bolivariano (2007), propone implementar metodologías de enseñanza novedosas que generen un aprendizaje significativo en el estudiante, que contribuyan al proceso de enseñanza y aprendizaje, y que tienen como base fundamental el perfil de un egresado con competencias, habilidades y destrezas idóneas exigidas en los estudios profesionales o superiores.

Partiendo de esta idea, es posible formar a un estudiante capaz de relacionarse con su contexto histórico-social, describir, interpretar, explicar, transformar y aprender de la realidad donde se produce el aprendizaje significativo. Dicho aprendizaje se logra cuando el nuevo contenido se relaciona sustancialmente con la 
estructura cognitiva del sujeto que aprende, haciéndolo capaz de elaborar sus propias ideas, y por lo tanto, de aplicar el nuevo conocimiento.

Por lo tanto, el compromiso de producir el cambio que se requiere, a través de la evaluación y reajustes en la forma de aprender, va a favorecer al grupo de estudiantes del nivel de educación básica primaria, con el objeto de facilitar el aprendizaje de los mismos, a lo que alude Montaigne, citado en Falieres y Antolin (2004): "El niño no es una botella que hay que llenar, sino un fuego que es preciso encender" (p. 69).

De tal manera, el estudiante es un participante activo en el proceso educativo de modo que, tiene la libertad de expresar sus dudas, inquietudes o comentarios al respecto del área en cuestión, manifestando una capacidad de análisis que despierte la curiosidad, atención e interés por la resolución de ejercicios de multiplicación de números naturales.

Cabe destacar a Colmenares (2013) quien plantea que el actual ejercicio del docente como gerente se ha caracterizado por la enseñanza tradicional, lo que conlleva en muchos casos a que los procesos educativos se conviertan en simples procesos de transmisión de información que limitan al estudiante la creatividad, la solución de problemas y la investigación.

Es por esta razón que el presente estudio propone diseñar un material didáctico basado en estrategias para el aprendizaje significativo, específicamente en el área de matemática de educación primaria. Así mismo, se considera que dichas estrategias de enseñanza y aprendizaje son el medio con que se logrará una transformación en los estudiantes con valores de solidaridad, compañerismo, cooperativismo y convivencia, permitiendo el entendimiento de los contenidos matemáticos.

Para lograrlo, la investigación se fundamentó en el estudio que realizó
Rodríguez (2013) donde develó la deficiencia en la aplicabilidad de estrategias lúdicas y la necesidad de diseñar dichas estrategias para el aprendizaje de los estudiantes. Aplicando las ideas de Rodríguez (2013) en la muestra de estudio, se pudo detectar la necesidad de aplicar este tipo de estrategias en los estudiantes de quinto grado de la Unidad Educativa Estadal "Aragüita" en el área de matemática.

Resulta pertinente mencionar en este estudio a Ausubel, Novak, y Hanesian, (1976) quienes afirman que la enseñanza por recepción o por descubrimiento "puede originar un aprendizaje memorístico o significativo lo cual va a estar definido por la forma en que los estudiantes adquieren la información siendo el docente quién opta por el enfoque para la enseñanza" (p.78). Visto de esta forma, el docente al formular o planificar algún tipo de ejercicios matemáticos, debe considerar el nivel de complejidad de cada uno de ellos, para que los estudiantes puedan comprenderlos más fácilmente.

De este modo, la tarea del docente se centra en disminuir las barreras del aprendizaje ya que el estudiante cuando se encuentra con ellas, se traslada a cualquier vía de escape bien sea: distracción, poca atención, poca tolerancia, falta de concentración o motivación, indisciplina, bajo rendimiento, entre otras. En este sentido, el docente es quien brinda al estudiante las herramientas, estrategias, metodología, para facilitar o guiar el logro de los aprendizajes.

Cabe destacar la importancia de dichos aspectos que son relevantes para el aprendizaje, y que son considerados en la investigación de Ausubel, Novak y Hanesian (1976), donde se afirma que la parte cognitiva y motivacional mejoran indudablemente el aprendizaje. De este modo, la retención y la trasferencia de las ideas que se aprenden por descubrimiento, así como la motivación, 
saberes previos, recepción y significatividad de los materiales de enseñanza tienen un impacto muy positivo en el aprendizaje. Es por ello que se considera presentar al estudiante los contenidos de forma que establezca una relación substancial y no de forma arbitraria, con ideas adecuadas a la capacidad humana para generar saberes, propiciando un ambiente ideal para que se produzca un aprendizaje significativo.

Por otra parte, el docente debe dejar claro o llevar al estudiante a concientizar y valorar la importancia que tienen las operaciones matemáticas en la vida, ya que ésta es una ciencia universal y aprenderla y conocerla le permite al estudiante adquirir habilidades y destrezas aplicables a otras áreas del saber. Por lo tanto, es necesario emplear materiales atractivos, diversos $y$ multidisciplinarios, para apoyar las clases y despertar así, el interés, la motivación y la actitud favorable en el estudiante, propiciar el análisis, interpretación y pensamiento lógico.

En este sentido, el desarrollo de esta investigación permitió analizar el aprendizaje por descubrimiento aplicado a la multiplicación de números naturales en los alumnos del quinto grado de la Unidad Educativa Estadal Aragüita. Cabe destacar el valioso sustento de estos autores para el tema abordado, Ausubel, Novak y Hanesian (1976), quienes exponen que "En el aprendizaje por descubrimiento el alumno debe descubrir este contenido por sí mismo, generando proposiciones que representen ya sea soluciones a los problemas planteados o los pasos sucesivos para resolverlos" (p. 64).

Por esta razón, el aprendizaje por descubrimiento conllevará al estudiante a relacionar los conocimientos previos que tenga sobre las operaciones matemáticas, con los que estará adquiriendo o descubriendo, lo cual permitirá que desarrolle una actitud positiva para la resolución de problemas, con mayor capacidad de entendimiento y lógica ante las diferentes circunstancias que se le presenten.
Visto desde esta perspectiva, la importancia de la presente investigación radica en que analiza el aprendizaje por descubrimiento en el área de matemáticas, específicamente en la multiplicación de números naturales. Para ello se tomó como objeto de estudio a los alumnos del quinto grado de la Unidad Educativa Estadal Aragüita, con el propósito de contribuir con su formación integral en el aprendizaje y desarrollo de habilidades en el área de matemáticas. También invita a los docentes a capacitarse en la metodología de enseñanza de las matemáticas, a actualizar sus conocimientos y habilidades con las nuevas tendencias a través del currículo, con el fin de brindar a los estudiantes una mejor formación y guiar el proceso educativo más eficazmente, produciendo un impacto muy positivo en el estudiante, ya que esto contribuirá a disminuir el alto porcentaje de niños y niñas con dificultades en esta área.

Para lograr el análisis del aprendizaje por descubrimiento antes mencionado, es importante conocer y detectarlas debilidades en el proceso de enseñanza para las matemáticas y particularmente la enseñanza para las operaciones de multiplicación de los alumnos. La indagación del tema fue de utilidad para llegar a detectar debilidades posibles en cuanto a la realización de los ejercicios de multiplicación de números naturales y, dado el caso, evaluar y analizar la aplicabilidad, incidencia y resultados del aprendizaje por descubrimiento con respecto a la enseñanza de la multiplicación de números naturales.

Por esta razón puede decirse que lo significativo de conducir esta investigación, radica en que establece posibles soluciones y recomendaciones ante la necesidad planteada y que serán de gran utilidad para propiciar un aprendizaje por descubrimiento en el estudiante. 
En vista de toda la problemática anteriormente planteada, y con la finalidad de buscarle una solución para satisfacer las necesidades señaladas, es que se procedió a formular la siguiente interrogante: ¿Cuál es la forma en que están recibiendo los estudiantes la información con respecto al contenido de la multiplicación de números naturales? La respuesta a esta interrogante permitió fundamentar un apoyo para mejorar la enseñanza de la multiplicación de números naturales, lo cual sirvió como el punto de partida para la consolidación de competencias en campos como la resolución de problemas, ejercicios matemáticos y además, para formar estudiantes con un gran sentido crítico, valorativo y con un próspero desarrollo del pensamiento lógico y analítico.

En relación a la interrogante formulada anteriormente, el objetivo general de esta investigación fue: analizar el aprendizaje por descubrimiento aplicado a la multiplicación de números naturales en los estudiantes del quinto grado de la Unidad Educativa Estadal Aragüita. Así mismo, los objetivos específicos se enfocaron en primer lugar en: diagnosticar el aprendizaje por descubrimiento aplicado a la multiplicación de números naturales, en segundo lugar, establecer el aprendizaje por descubrimiento aplicado a la multiplicación de números naturales a la luz de la teoría de Ausubel y finalmente, determinar el aprendizaje por descubrimiento en la realización de la multiplicación de números naturales.

Ahora bien, para efectos de la presente investigación, partiendo de la idea de que el aprendizaje por descubrimiento aplicado a la multiplicación de números naturales se considera es unos de los tópicos que se imparten en el aula, el aprendizaje significativo que plantea Ausubel, se materializa cuando las tareas que se le asignan al estudiante están relacionadas de manera congruente con el mismo, y por lo tanto, aumenta su interés en el saber.

De este modo, el aprendizaje significativo se logra cuando el estudiante como constructor de su propio conocimiento relaciona los conceptos a aprender y les da un sentido a partir de la estructura conceptual que ya posee, es decir, sus conocimientos previos. De este modo, el estudiante construye nuevos conocimientos a partir de los que ya ha adquirido anteriormente. Este aprendizaje puede ser por descubrimiento o receptivo. Pero además lo logra porque quiere, es decir, está interesado en aprender.

Por lo anteriormente expuesto, se puede decir que esta investigación está justificada por la necesidad de resolver o al menos minimizar, la dificultad que presentan numerosos estudiantes en lo referente a la resolución de los ejercicios de multiplicación de números naturales en el quinto grado de la Segunda Etapa de Educación Básica de la Unidad Educativa Estadal Aragüita; dificultad que se evidencia principalmente en el dominio de la tabla de multiplicar, como factor o condición primordial para resolver este tipo de operaciones.

\section{MÉTODO}

La presente investigación se caracterizó por la premisa del paradigma positivista con enfoque cuantitativo, el cual considera los acontecimientos reales, no solo desde su naturaleza misma, sino también de los datos que proporcionan la observación y la experimentación. Se ubicó en un diseño de investigación no experimental, considerando que este se realiza sin manipular en forma deliberada ninguna variable. Al respecto, Palella y Martins (2010) afirman que, en este caso, "El investigador no sustituye intencionalmente las variables independientes. Se observan los hechos tal y como se presentaron en su contexto real y en un tiempo 
determinado o no, para luego analizarlos". (p.87)

Aunado a esto se consideró como muestra de estudio a los estudiantes del quinto grado, de la Unidad Educativa Estadal Aragüita, en el hecho real donde se evidenciaron las dificultades que tenían en cuanto a la realización de ejercicios de multiplicación de números naturales. Estos acontecimientos fueron observados y analizados tal y como se presentaron en el aula de clase.

En este orden de ideas, el estudio fue definido como una investigación de campo porque se recolectaron los datos: "directamente de la realidad donde ocurren los hechos sin manipular o controlar variables". (Palella y Martins, 2010 p. 88). De este modo, se utilizaron datos visibles para el investigador, tales como: fuentes de información, análisis, procesamiento y dilucidación, proporcionando así el desarrollo y logro de los objetivos propuestos.

De allí, se describió la situación real en el aula del quinto grado, tratando específicamente la dificultad que presentaron los estudiantes en el aprendizaje de la multiplicación de números naturales. Se buscó discernir en los aspectos o factores que incidieron en la dificultad planteada con el objetivo de enmarcar dentro de las conclusiones, recomendaciones, respuestas o aportes para el abordaje del problema en cuestión.

Como se dijo anteriormente, para el desarrollo de esta investigación, fue considerada la Unidad Educativa Estadal "Aragüita", ubicada en la Ceiba, un sector de Guacara, Estado Carabobo, Venezuela. Se trata de una escuela pública, con una estructura pequeña donde se detectó que los estudiantes tenían una actitud de incertidumbre y desconocimiento en los procesos matemáticos.

En este sentido, se estimó para muestra no probabilística a 26 estudiantes del quinto grado de dicha institución, a quienes se les aplicó una lista de cotejo conformada por 17 ítems, a fin de determinar el aprendizaje por descubrimiento aplicado a la multiplicación de números naturales.

Por consiguiente, se desarrolló preliminarmente la técnica de observación directa en el aula con los estudiantes de muestra donde se captó la realidad existente en la misma, que fue plasmada en un registro anecdótico que luego de ser analizado, constató la necesidad de llevar a cabo la intencionalidad de esta investigación.

Puesto que, para conducir este estudio, y con el fin de obtener los datos requeridos para determinar el nivel de dificultad en cuanto al aprendizaje por descubrimiento en este caso, de la multiplicación de números naturales, fue necesaria la aplicación de un instrumento de medición cuantitativa, por lo cual se tomó en cuenta a Hernández, Fernández y Baptista (2010), quienes sostienen que "un instrumento de medición adecuado es aquel que registra datos observables que representan verdaderamente a los conceptos o variables que el investigador tiene en mente". (p. 200)

Por esta razón se empleó una lista de cotejo formulada para dar respuestas a 17 ítems cerrados dicotómicos cuya opción de respuesta fue Si y No, en el que se adquirió información acerca del nivel o grado de conocimiento de los estudiantes en la multiplicación de números naturales. Con esta lista se obtuvieron datos relevantes sobre los sujetos directos que se involucraron en la investigación a fin de analizar el aprendizaje por descubrimiento aplicado a la multiplicación de números naturales.

En cuanto a la validación del instrumento empleado, se solicitó su revisión a tres expertos altamente calificados, los cuales lo revisaron exhaustivamente. La confiabilidad se realizó mediante un estudio piloto para su comprobación preliminar. Esto permitió saber si el instrumento requería alguna modificación o perfeccionamiento en su estructura formal. 
También permitió saber el grado de dificultad de los ítems, exactitud, consistencia y precisión, es decir, los aspectos que debe poseer todo instrumento de medición. Para establecer el grado de confiabilidad del instrumento, se utilizó el Coeficiente $\mathrm{KR}_{20}$ de Kuder-Richardson, donde se obtuvo una confiabilidad de 0,85. Esto quiere decir, 85 de cada 100 veces que se aplique el instrumento, los resultados serán idénticos.

Ahora bien, para el desarrollo de esta investigación, se cumplieron las siguientes fases: en primer término, se realizó un diagnóstico del nivel de aprendizaje por descubrimiento a través una lista de cotejo. Posteriormente, se procedió con el análisis de los resultados en contraste con las teorías de aprendizaje significativo de David Ausubel, Motivación de Abraham Maslow y la teoría del Aprendizaje de Vygotsky.

Todos estos fundamentos proporcionaron valiosos aportes en lo referente a estrategias y formas de impartir el conocimiento, facilitó la comprensión del investigador en torno a la actitud de los estudiantes en la resolución de ejercicios de multiplicación, detectar debilidades en la forma como el docente enseña la multiplicación de números naturales $y$, una serie de aspectos que fundamentaron la razón de ser de cada ítem.

\section{RESULTADOS}

Ahora bien, haciendo un contraste con los fundamentos teóricos los cuales sustentaron esta investigación, es decir, las teorías de aprendizaje significativo de Ausubel, se exponen a continuación los ítems más relevantes.

En cuanto al ítem 1, se evidencia que el cien por ciento $(100 \%)$ de los que presentaron la prueba (26 estudiantes) contestaron $\mathrm{Si}$ al saber identificar el símbolo utilizado para la multiplicación, tal como se representa en el Gráfico 1.

SI: $100 \%$

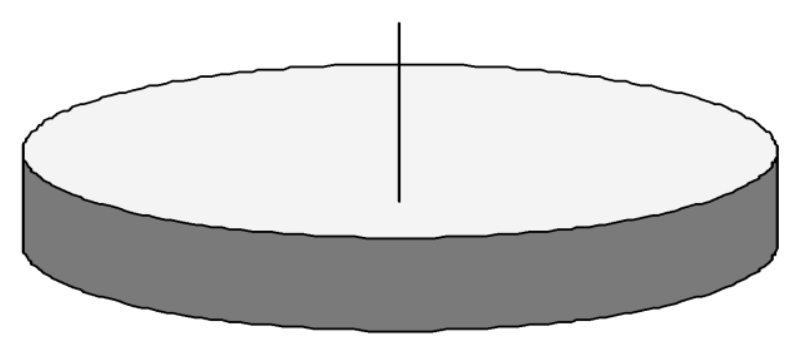

Gráfico 1. Identificación del símbolo utilizado para la multiplicación. (Fuente: Barrios y Camacho, 2020)

El análisis de este primer ítem concuerda con Ausubel, Novak, y Hanesian, (1976), en su primera aproximación a la teoría del aprendizaje significativo. En esta etapa la esencia del proceso del aprendizaje reside en que las ideas expresadas simbólicamente son relacionadas de modo sustancial con lo que el alumno ya sabe. Por relación sustancial y no arbitraria se quiere decir que las ideas se relacionan con algún aspecto específico y relevante existente en la estructura cognoscitiva del alumno como lo puede ser, una imagen, un símbolo, un concepto o una proposición. En este proceso se busca que el estudiante establezca relaciones entre los nuevos contenidos y los que ya posee y de este modo logre un aprendizaje significativo más que memorístico en la multiplicación de números naturales. 
Analizando las respuestas facilitadas al planteamiento 2, se evidencia a través del Gráfico 2, que el 85 por ciento de los encuestados (22 estudiantes) contestó que
No tienen conocimientos de la tabla de multiplicar, mientras que el 15 por ciento restante de los encuestados (4 estudiantes) respondió que Si lo tenía.

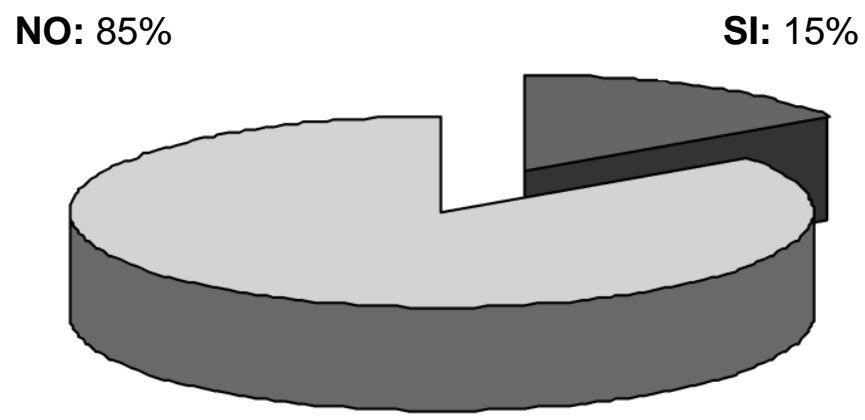

Gráfico 2. Tiene conocimientos de la tabla de multiplicar. (Fuente: Barrios y Camacho, 2020)

Esto corrobora lo planteado por Ausubel, Novak, y Hanesian, (1976), donde aprender es sinónimo de comprender, lo que implica tener una visión del aprendizaje basada en los procesos internos del alumno y no solo en sus respuestas externas. Con la intención de promover la asimilación de los saberes, el profesor utilizará organizadores previos que favorezcan la creación de relaciones adecuadas entre los saberes previos y los nuevos. Los organizadores tienen la finalidad de facilitar la enseñanza receptivo significativa, con lo cual, sería posible considerar que la exposición organizada de los contenidos, propicia una mejor comprensión.

Seguidamente, analizando las respuestas aportadas en el ítem 8, en el Gráfico 3 se observa que el 54 por ciento de los encuestados (14 estudiantes) contestó que No tenían interés y seguridad al momento de realizar los ejercicios de multiplicación de números naturales, mientras que el 46 por ciento restante de los encuestados (12 estudiantes) respondió que Si los tenían.
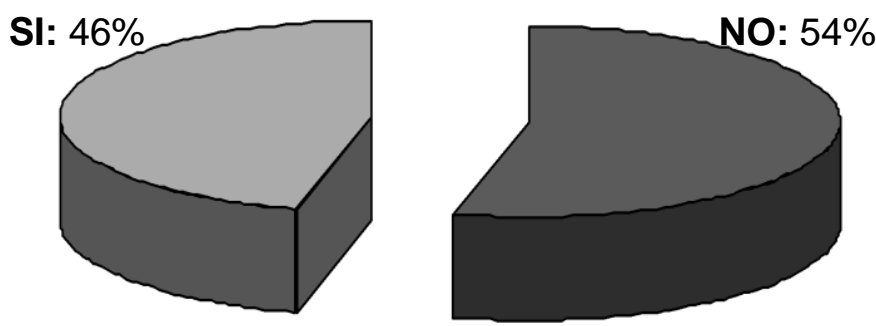

Gráfico 3. Interés y seguridad al momento de realizar los ejercicios. (Fuente: Barrios y Camacho, 2020) 
Para el análisis de este resultado se toma en cuenta la teoría de Maslow (1991) y su estudio sobre la autorrealización y las necesidades del ser, que se clasifican en inferiores y superiores. En el caso de las necesidades del nivel superior el autor dice que estas son de naturaleza intelectual, de apreciación estética y autorrealización. De este modo cuando éstas son satisfechas, la motivación de las personas no cesa, al contrario, aumenta para conseguir mayores logros, es decir, cuanto más éxito obtiene de sus esfuerzos por conocer, más se empeñará en conseguir más conocimientos. A diferencia de las necesidades por deficiencia, que nunca pueden satisfacerse por completo y la motivación de alcanzarlas se renueva de modo interminable.

Dicho autor realiza una jerarquización de las necesidades, la cual va a definir la motivación como una herramienta para el logro de metas dentro de una institución educativa.
Con esto se va a permitir superar cada uno de los aspectos que condicionan en algunos casos el buen desempeño del docente. Para lograrlo, es importante el empleo de estrategias motivacionales considerando la jerarquización de las necesidades expuestas por Maslow, es decir, las necesidades sociales como la aceptación, sentido de pertenencia $\mathrm{y}$ afecto; las necesidades de estima específicamente de autonomía, logro y reconocimiento; y finalmente la autorrealización.

Seguidamente, al analizar si utiliza procedimientos lógicos para la realización de los ejercicios, en el ítem 12, se evidencia que el 62 por ciento de los encuestados (16 estudiantes) respondieron que $\mathrm{Si}$ utilizaron procedimientos lógicos para la realización de los ejercicios de multiplicación de números naturales, mientras que el 38 por ciento restante de los encuestados (10 estudiantes) aseveró que No los utilizaban. Esto se puede observar en el siguiente gráfico.

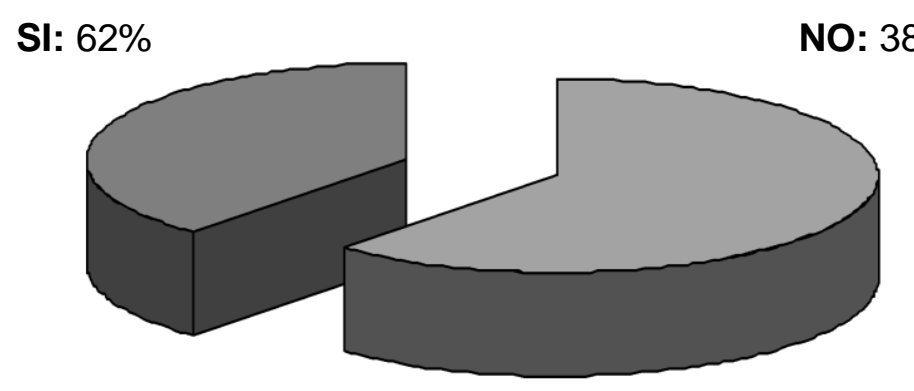

Gráfico 4. Ordena correctamente las cantidades para realizar los ejercicios. (Fuente: Barrios y Camacho, 2020)

En este ítem está presente la significatividad lógica que según Ausubel, Novak, y Hanesian, (1976) es una de las circunstancias que deben darse para que se produzca el aprendizaje significativo. Dicho ítem hace referencia a la estructura interna del contenido, manteniendo la responsabilidad del docente de guiar los procesos de aprendizaje eficazmente. De aquí se considera necesario desarrollar en el estudiante, un pensamiento lógico matemático ante la presencia del conocimiento facilitado por el docente, sin obviar que hay una lógica interna que le ayuda a resolver los ejercicios planteados. Esto quiere decir, en pocas palabras, que el estudiante no solo puede construir su conocimiento a través de instrumentos metodológicos sino a partir de su propia experiencia. 
En cuanto al ítem 17, el 100 por cien de los encuestados (26 estudiantes) resaltó que No tomaron decisiones acertadas ante un procedimiento equívoco como se puede apreciar en el Gráfico 4. Por esta razón lo que sugiere la investigación es aplicar estrategias de trabajo en equipos u otra técnica didáctica donde los estudiantes puedan trabajar de forma grupal, se generen situaciones de intercambio de conocimientos, conversaciones, sugerencias, opiniones, procurando que aquellos en su totalidad que presentaron fallas, con la orientación del docente puedan despejar sus dudas y aprender.

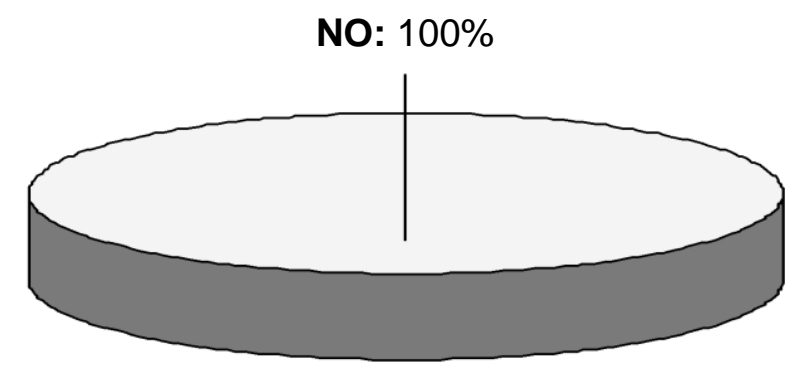

Gráfico 5. Habilidad de identificar desaciertos. (Fuente: Barrios y Camacho, 2020)

En esta parte, es preciso destacar lo señalado por Vygotsky (1979) "a mayor interacción social, mayor conocimiento, más posibilidades de actuar, más robustas funciones mentales" (p.28). Es decir, mientras más rica y frecuente sea la interacción con los demás, el conocimiento será más rico y amplio. Por consiguiente, la zona de desarrollo próximo, consecuentemente, está determinada socialmente y se aprende con la ayuda de los demás, en el ámbito de la interacción social.

\section{CONCLUSIONES}

En primera instancia, luego de realizado el diagnóstico inicial a los estudiantes del quinto grado de la Unidad Educativa Estadal "Aragüita", en relación al aprendizaje por descubrimiento, se logró así determinar el que en el estudio de la multiplicación de números naturales se evidencia la necesidad de aplicar la metodología planteada en esta investigación.

También, se pudo analizar la problemática en torno al aprendizaje por descubrimiento aplicado a la multiplicación de números naturales. En este análisis se concluyó que dicha situación tiene que ver con debilidades en las estrategias de enseñanza aprendizaje, vacíos de contenidos, falta de interés, motivación, entre otros aspectos. En general, un desconocimiento total de la forma idónea para impartir el conocimiento de manera significativa y eficazmente, sobre todo en el área de matemática, donde algunos contenidos parecen abstractos, por lo que es pertinente dotar a los estudiantes de recursos que les permita relacionar esos contenidos con el quehacer diario.

Aunado a esto, se pudo detectar que los estudiantes carecían de conocimientos previos respecto al área en cuestión, es decir, desconocían las tablas de multiplicar y el proceso de la multiplicación en sí, por lo cual, durante las actividades de aula, no estaban presentes cognitivamente. Es posible decir que esta dificultad se venía arrastrando de niveles anteriores, aspecto relevante para entender las razones por las cuales los estudiantes no resolvieron los ejercicios de forma satisfactoria. 
Así mismo, se pudo observar que el docente está al tanto de la dificultad que presentaron los estudiantes en la solución de los ejercicios de multiplicación de números naturales. También es consciente de que esta dificultad la traen consigo de grados anteriores. En pocas palabras, los profesores saben que hay una deficiencia en el área de matemática y por lo tanto, se deben tomar medidas para abordar esta situación.

De igual manera, el proceso de enseñanza aprendizaje que se da en el aula, se limita a un patrón tradicional, donde por lo general el estudiante asume un papel pasivo durante el proceso. Por esta razón, se hace un llamado a rediseñar las estrategias basadas en teorías de aprendizaje por descubrimiento que propicien en el estudiante un desarrollo del pensamiento crítico, el razonamiento lógico y la contextualización del conocimiento de modo que, todo lo que se le enseña en la escuela pueda ser aplicado en su cotidianidad.

Aunado a esto, hay que valorar también el desempeño del docente de aula como facilitador y mediador del aprendizaje significativo, lo cual es un elemento esencial para la materialización de los objetivos proyectados en el nuevo currículo y la consecución de la misión y la visión trazada por el Estado venezolano, como ente rector de la educación en la nación. De hecho, la información y procedimientos antes analizados que sustentan esta investigación, demuestran la necesidad de analizar el aprendizaje por descubrimiento aplicado al contenido matemático, específicamente en la multiplicación de números naturales.

Es conveniente recalcar en estas conclusiones, que también se logró establecer una serie de criterios operativos con respecto a otros campos de la teoría del aprendizaje por descubrimiento, en este caso, aplicado a la enseñanza de la multiplicación de números naturales. Estos criterios fueron planificados de acuerdo con los intereses de quienes van a enseñar y de quienes van a aprender. En consecuencia, se obtuvieron resultados muy positivos, significativos y satisfactorios, gestando estudiantes perseverantes, capaces de salir adelante ante cualquier situación, sobre todo considerando que la dinámica social actual ha generado la necesidad casi inmediata de ingeniarse 0 descubrir procedimientos que faciliten los procesos y sistemas.

Se recomienda a los docentes, directivos, planificadores y a todos los participantes del proceso educativo, promover la resolución de problemas prácticos que propicien un aprendizaje por descubrimiento en el área de matemática. Así mismo, también se recomienda extrapolar una gama más amplia de problemas prácticos, considerando además los procesos de interacción social como base fundamental en el desarrollo y el aprendizaje de los estudiantes.

Para finalizar, se puede decir que el aprendizaje por descubrimiento se puede aplicar como respuesta al problema de deserción escolar en el área de matemática. Sin embargo, por la amplitud que abarca esta teoría de aprendizaje se puede emplear en otras áreas de estudio por la sencilla razón de que, de todos los conocimientos que adquiere el ser humano a lo largo de su vida, prevalecen y trascienden aquellos que utiliza y aprovecha en su desarrollo personal, cotidiano y profesional.

\section{REFERENCIAS}

Álvarez, Y. (2006). ¡Auxilio! No puedo con la matemática. Revista Iberoamericana de Educación Matemática Equisángulo, 2(1), 4-16

Ausubel, D. P., Novak, J. D., y Hanesian, H. (1976). Psicología educativa: un punto de vista cognoscitivo (Vol. 3). México: Trillas

Colmenares, Y. (2013). Material Didáctico Basado en Estrategias de Aprendizaje Significativo para la Efectividad de la Gestión del Conocimiento en el Área de 
Matemática de Educación Primaria. (Tesis de maestría inédita). Facultad de Ciencias de la Educación, Universidad de Carabobo. Valencia, Venezuela

Delors, J. (1996). Informe a la UNESCO de la comisión internacional sobre la educación para el siglo XXI. La educación encierra un tesoro. Recuperado de:http://biblioteca.uazuay.edu.ec/opac css/index.php?|vl=notice_display\&id $=47$ 802. [Consultado: 10 de enero, 2017]

Falieres, N. y Antolín, M. (2004). Como mejorar el aprendizaje en el aula y poder evaluarlo. Argentina: Círculo latino austral grupo CLASA

Hernández, R., Fernández, C. y Baptista, P. (2010). Metodología de la investigación. (5a ed.) México: McGraw-Hill

Ley Orgánica de Educación. Gaceta Oficial de la República de Venezuela, 5.929 (Extraordinario), Agosto 15, 2009

Maslow, A. (1991). Motivación y personalidad. Ediciones Díaz de Santos

Ministerio de Educación, Cultura y Deporte. (2007). Currículo del Subsistema de
Educación Primaria Bolivariana. Caracas, Venezuela: Fundación Centro Nacional para el Mejoramiento de la Enseñanza de la Ciencia, CENAMEC

Pallela, S. y Martins, F. (2010). Metodología de la Investigación Cuantitativa. ( $3^{\mathrm{a}}$ ed.) Caracas: Venezuela. Edición: FEDUPEL

Rodríguez, E. (2013), Estrategias Lúdicas para el aprendizaje de los estudiantes de educación básica en la E.B.N "José Gregorio Guitian. (Tesis de maestría inédita). Facultad de Ciencias de la Educación, Universidad de Carabobo. Valencia, Venezuela

Sánchez, G. (2010). Estrategias aplicadas por los docentes y su relación con el aprendizaje matemático de los alumnos de segundo año del sub-sistema educación secundaria del liceo bolivariano "Cirilo Alberto". (Tesis de maestría inédita). Facultad de Ciencias de la Educación, Universidad de Carabobo. Valencia, Venezuela

Vygotsky, L. (1979). El desarrollo de los procesos psicológicos superiores. Barcelona: Grijalbo 